\title{
Comparison of four algorithms on establishing continuous reference intervals for pediatric analytes with age-dependent trend
}

\author{
Kun Li ${ }^{1,2}$, Lixin $\mathrm{Hu}^{3}$, Yaguang Peng ${ }^{2}$, Ruohua Yan², Qiliang Li $i^{3}$ Xiaoxia Peng ${ }^{1,2^{*}}$, Wenqi Song ${ }^{3^{*}}$ and Xin $\mathrm{Ni}^{2,4^{*}}$
}

\begin{abstract}
Background: Continuous reference intervals (RIs) allow for more precise consideration of the dynamic changes of physiological development, which can provide new strategies for the presentation of laboratory test results. Our study aimed to establish continuous Rls using four different simulation methods so that the applicability of different methods could be further understood.

Methods: The data of alkaline phosphatase (ALP) and serum creatinine $(\mathrm{Cr})$ were obtained from the Pediatric Reference Interval in China study (PRINCE), in which healthy children aged 0-19years were recruited. The improved non-parametric method, the radial smoothing method, the General Additive Model for Location Scale and Shape (GAMLSS), and Lambda-Median-Sigma (LMS) were used to develop continuous Rls. The accuracy and goodness of fit of the continuous Rls were evaluated based on the out of range (OOR) and Akaike Information Criterion (AIC) results.

Results: Samples from 11,517 and 11,544 participants were used to estimate the continuous RIs of ALP and Cr, respectively. Time frames were partitioned to fulfill the following two criteria: sample size $=120$ in each subgroup and mean difference $=2$ between adjacent time frames. Cubic spline or penalized spline was used for curve smoothing. The Rls estimated by the four methods approximately overlapped. However, more obvious edge effects were shown in the curves fit by the non-parametric methods than the semi-parametric method, which may be attributed to insufficient sample size. The OOR values of all four methods were smaller than $10 \%$.
\end{abstract}

Conclusions: All four methods could be used to establish continuous RIs. GAMLSS and LMS are more reliable than the other two methods for dealing with edge effects.

Keywords: Continuous reference intervals, Pediatric, Graphical report, Clinical laboratory

\footnotetext{
* Correspondence: pengxiaoxia@bch.com.cn; songwenqi1218@163.com; nixin@bch.com.cn

'Department of Epidemiology and Health Statistics, School of Public Health, Capital Medical University, Beijing 100069, China

${ }^{3}$ Department of Clinical Laboratory Center, Beijing Children's Hospital, Capital Medical University, National Center for Children Health, No.56 Nanlishi Road, Beijing 100045, China

${ }^{2}$ Center for Clinical Epidemiology and Evidence-based Medicine, Beijing Children's Hospital, Capital Medical University, National Center for Children Health, No.56 Nanlishi Road, Beijing 100045, China

Full list of author information is available at the end of the article
}

\section{Background}

Reference intervals (RIs) are commonly used in medicine to interpret laboratory test results and have been traditionally used in clinical practice to aid diagnosis [1]. Pediatric RIs represent the physiological conditions of normal children and adolescents during development [2]. Dynamic anatomical and physiological development accounts for high variability of many biochemical

(C) The Author(s). 2020 Open Access This article is licensed under a Creative Commons Attribution 4.0 International License, which permits use, sharing, adaptation, distribution and reproduction in any medium or format, as long as you give appropriate credit to the original author(s) and the source, provide a link to the Creative Commons licence, and indicate if changes were made. The images or other third party material in this article are included in the article's Creative Commons licence, unless indicated otherwise in a credit line to the material. If material is not included in the article's Creative Commons licence and your intended use is not permitted by statutory regulation or exceeds the permitted use, you will need to obtain permission directly from the copyright holder. To view a copy of this licence, visit http://creativecommons.org/licenses/by/4.0/. The Creative Commons Public Domain Dedication waiver (http://creativecommons.org/publicdomain/zero/1.0/) applies to the data made available in this article, unless otherwise stated in a credit line to the data. 
analytes with increasing age, particularly in the first years of life and during puberty [3]. Therefore, the definitions of pediatric RIs should consider special population features, among which age and sex are the most important for children and adolescents [4].

A familiar method to elucidate trends of age dependence of biochemical analytes is to establish RIs for each age partition. The use of discrete RIs for different age groups is well-established in clinical practice and allows easy integration into current laboratory information systems. To improve the accuracy of age partitioning, an age partitioning algorithm for RI estimation was developed in our previous publication [2]. However, it is still difficult for that model to describe analyte concentrations at the margins of age partitioning, especially abrupt changes during relatively narrow age periods, such as a radical decrease of alkaline phosphatase (ALP) during puberty [5]. Further, it may be difficult to obtain suitable age partitioning points for analytes with continuous upward trends, such as serum creatinine $(\mathrm{Cr})$.

Analogous to other developmental quantities whose relationships with age are routinely analyzed, a continuous description would seem to be more appropriate for laboratory analytes with special age-dependent trends [6]. For example, growth curves were used by the World Health Organization (WHO) to construct child growth standards [7]. The current approaches for establishing continuous RIs can be divided into the non-parametric and semi-parametric method $[3,5,6,8,9]$. These different statistical methods of curve simulation could produce different RIs using the same data [1]. Therefore, it is imperative to explore which method is the most appropriate for RI estimation of analytes with various agedependent trends. To our knowledge, few statistical simulations have been reported to evaluate how well these methods estimate continuous RIs.

Our aim in the present study is to compare the accuracy of continuous RIs established using four different curve simulation methods to better understand these methods' applicability. The continuous RIs could facilitate the generation of graphical reports in clinical laboratory settings, which could provide quantitative and dynamic assessments of laboratory test results instead of only absolute values [6].

\section{Methods}

\section{Data source}

Data were obtained from the results of the PRINCE study. The eligibility criteria and other detailed information were previously published [10]. In brief, 14,646 healthy children aged 0-19 years were recruited from the northeast (Liaoning Province), north (Beijing Municipality and Hebei Province), northwest (Shaanxi Province), middle (Henan and Hubei provinces), south
(Guangdong Province), southwest (Chongqing Municipality and Sichuan Province), and east (Shanghai Municipality and Jiangsu Province) regions of China from January 2017 to August 2018. All participants were confirmed to be eligible based on a questionnaire screening and subsequent physical examination. Considering that the sample size of children aged less than 1 year was limited, we only included healthy children aged $1-19$ years. Analyte tests were measured on a Cobas C702 automated biochemistry analyzer (Roche Diagnostics $\mathrm{GmbH}$, Mannherim, Germany) at the Department of Clinical Laboratory Center of Beijing Children's Hospital, which was the central laboratory of the PRINCE study. Detailed information on quality control was described in the published protocol [10]. The ALP and Cr analytes were selected from 13 eligible biochemical markers as typical cases because of their special age-dependent trends in children and adolescents. The study was exempted by the Ethics Committee of Beijing Children's Hospital, affiliated with Capital Medical University, Beijing, China.

\section{Data cleaning and management}

Data cleaning was performed to detect missing values and outliers. Missing values were defined as incomplete information of age, sex, or biochemical analytes. Considering that ALP and $\mathrm{Cr}$ are known to vary significantly by age and sex [6], outliers are removed according to sex and age groups (for each 1-year) by Tukey's method [4]. In this method, outliers are removed if they are less than $\mathrm{Q}_{1}-1.5 \times \mathrm{IQR}$ or more than $\mathrm{Q}_{3}+1.5 \times \mathrm{IQR}$, in which $\mathrm{Q}_{1}$ and $\mathrm{Q}_{3}$ are the 25th and 75th percentiles, respectively. IQR is interquartile range, calculated by $Q_{3}-Q_{1}$, where the data have a Gaussian distribution. Otherwise, the data should be transformed by the Box-cox method, expressed by the following formula:

$$
y=\left\{\begin{array}{lc}
\left(\mathrm{x}^{\lambda}-1\right) / \lambda & \text { for } \lambda \neq 0 \\
\ln (\mathrm{x}+\mathrm{c}) & \text { for } \lambda=0
\end{array}\right.
$$

where $x$ is the original value, $y$ is the value after Box-cox transformation, and $\lambda$ and $c$ are parameters calculated by maximum likelihood estimation.

\section{Statistical simulations}

All statistical analysis was performed using SAS 9.4 and $\mathrm{R}$ 3.5.1. The lower limit and upper limit values of RIs were calculated as the 2.5 and $97.5 \%$ quantiles of the corresponding populations, respectively. Four methods were implemented in this study: the improved nonparametric method, the radial smoothing method (RS), the General Additive Model for Location Scale and Shape method (GAMLSS), and the Lambda-MedianSigma method (LMS) [4, 8, 11-13]. Both the improved non-parametric method and RS are considered as non- 
parametric methods because non-parametric curve estimation methods are used during the RI establishment procedure. Although GAMLSS and LMS use smoothing methods in model terms, they are deemed semiparametric methods because the response variable requires the assumption of a parametric distribution.

In the past decades, several studies have used spline or piecewise polynomial methods to establish continuous RIs of laboratory analytes for interpretation of the age dynamics of children's development [5, 6, 14]. These studies' methods can be divided into three steps. First, the whole dataset was split into several age groups; then, discrete RIs were calculated for each age group; finally, the RIs' limit values for each age group were fit using appropriate smoothing methods, such as spline or polynomial methods $[4,6]$. Arzideh et al. optimized the age group classification procedure $[3,15]$. They split the whole dataset into overlapping time frames, which allows more precise consideration of rapid changes in analyte concentrations with increasing age. We call Arzideh's method the improved non-parametric method in the present study and used the bootstrap method to calculate the reference limits for each time frame $[3,16]$. To find the most suitable smoothing method for the improved non-parametric method, cubic spline, penalized spline, and fractional polynomial smoothing were performed, and the goodness of fit was evaluated by Akaike information criterion (AIC) values calculated under each model [17]. The formula for AIC is as follows.

$$
A I C=-2 \operatorname{lon} \hat{\theta}+k
$$

where $\hat{\theta}$ is the maximized log likelihood function, and $k$ is the number of effective degrees of freedom used in the model, e.g., $k=2$. The model with the smallest AIC value is considered to have the best fit.

The LMS model contains three parameters: skewness (L) accounts for deviation from the normal distribution after Box-Cox transformation; the median (M) models the outcome variable depending on one explanatory variable; and the coefficient of variation (S) accounts for variation of data points around the mean and adjusts for non-uniform dispersion [9]. GAMLSS is an extension of the LMS method, which was introduced by Rigby and Stasinopoulos as a way of overcoming some of the limitations associated with generalized linear models and generalized additive models $[11,18]$. In contrast with LMS, GAMLSS can accommodate more than one covariate and distribution $[11,19]$. The Box-Cox $t$ and BoxCox power exponential distributions were compared to select the most appropriate type of GAMLSS model [20, 21]. Worm plots were used to assess the fitting results of additive terms and to judge whether simulation of kurtosis was required [22]. The procedure was implemented in the GAMLSS package (version 5.1-2) of the R statistical software package.

In the RS method, various spline functions, such as Bspline and truncated polynomial functions, can be used as the basis function to fit non-parametric curve estimation [8]. Radial bases are sometimes preferred for higher-dimensional problems because of their straightforward extension. Wan $\mathrm{XH}$ et al. provided a percentile curve for calculation of arithmetic based on four moments and the Edgeworth-Cornish-Fisher expansion, which was used for some of the present study's simulations [23].

Before statistical simulation, the whole dataset was randomly partitioned into training and test datasets in an 8:2 ratio. The training dataset were used for model fitting and model selection, and the test dataset were used for assessment of the model's predictive power to fit training data, i.e., the out of range (OOR) percentage. Considering sex differences in analyte concentrations, the data were divided by sex before the training and test datasets were generated. The process of data set partitioning and RI calculation was repeated 100 times for both ALP and Cr to reduce the random error caused by running too few statistical simulations. In addition, the Wilcoxon test was used to compare whether the training and test datasets had similar age distributions. When $P \geq 0.05$, the results of dataset partitioning were deemed valid, and otherwise, the partitioning procedure was repeated. According to the recommendations of the Clinical and Laboratory Standards Institute, RIs may be considered valid when the OOR value is $<10 \%$ [4], considering that we estimated RIs using 95\% intervals. Thus, OOR values close to $2.5 \%$ for both the upper and lower reference limits were appropriate for method selection. Furthermore, AIC values were calculated to evaluate the different models' goodness of fit under the GAMLSS method. Then, the accuracy and goodness of fit of continuous RIs were compared comprehensively based on the OOR and AIC values. The statistical simulation process is summarized in Supplemental Figure 1.

\section{Results}

\section{Characteristics of ALP and $\mathrm{Cr}$ distributions}

The entire data cleaning process is shown in Fig. 1. Scatter diagrams show outliers that were removed by Tukey's method (Supplemental Figure 2). After data cleaning, samples from 11,517 and 11,544 participants aged 1-19 years were included to calculate the RIs of ALP and Cr, respectively. The distributions of ALP and $\mathrm{Cr}$ by age and sex are shown in Table 1 . The probability density plots had the same age distributions between the training and test datasets (Supplemental Figure 3).

We represented the density of the data points by color chromaticity using the plotSimpleGamlss function in $\mathrm{R}$. 


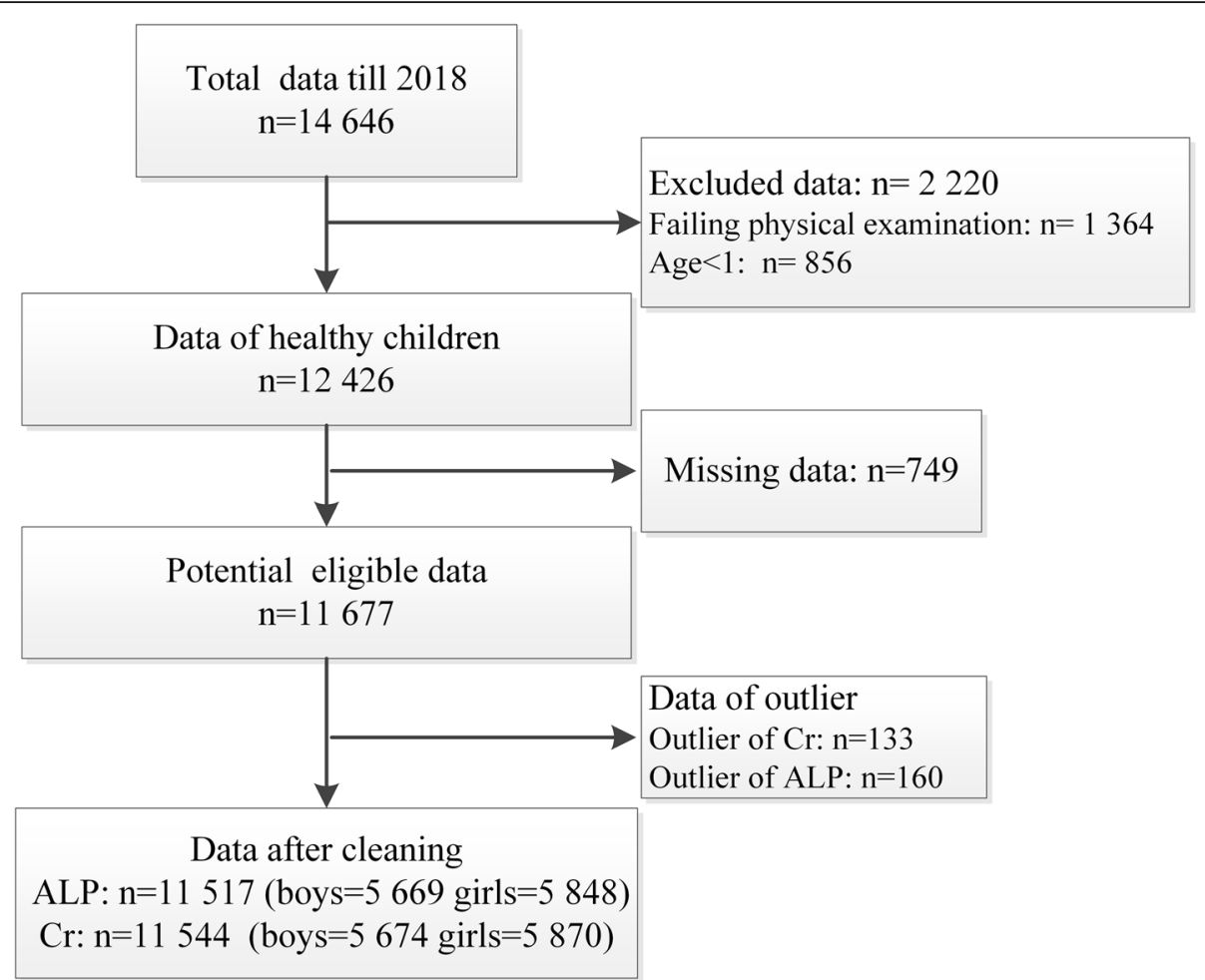

Fig. 1 Data cleaning procedure. ALP, alkaline phosphatase; $\mathrm{Cr}$, serum creatinine

Table 1 The distributions of alkaline phosphatase and serum creatinine by age and sex

\begin{tabular}{|c|c|c|c|c|c|c|c|c|c|c|c|c|}
\hline \multirow{3}{*}{$\begin{array}{l}\text { Age } \\
\text { (years) }\end{array}$} & \multicolumn{6}{|c|}{ Alkaline phosphatase (U/L) } & \multicolumn{6}{|c|}{ Serum creatinine (umol/L) } \\
\hline & \multicolumn{3}{|c|}{ Boys } & \multicolumn{3}{|l|}{ Girls } & \multicolumn{3}{|c|}{ Boys } & \multicolumn{3}{|l|}{ Girls } \\
\hline & $n$ & Mean & SD & $n$ & Mean & SD & $n$ & Mean & SD & $n$ & Mean & SD \\
\hline$>=1$ & 255 & 253.96 & 62.55 & 225 & 243.68 & 50.98 & 260 & 24.85 & 3.52 & 227 & 23.78 & 3.33 \\
\hline $2 \sim$ & 304 & 228.59 & 47.73 & 266 & 236.15 & 55.18 & 309 & 28.58 & 4.02 & 273 & 28.61 & 4.46 \\
\hline $3 \sim$ & 446 & 222.06 & 45.83 & 337 & 220.36 & 45.17 & 449 & 32.61 & 4.23 & 328 & 31.08 & 3.50 \\
\hline 4 & 287 & 214.88 & 42.28 & 298 & 224.49 & 47.75 & 286 & 35.14 & 4.36 & 300 & 34.24 & 4.34 \\
\hline 5 & 286 & 226.13 & 47.68 & 280 & 233.63 & 47.95 & 289 & 37.18 & 4.32 & 284 & 36.95 & 4.50 \\
\hline 6 & 393 & 227.92 & 43.78 & 355 & 230.68 & 47.52 & 392 & 40.90 & 5.34 & 362 & 40.02 & 4.99 \\
\hline 7 & 413 & 231.92 & 52.08 & 372 & 237.97 & 51.65 & 409 & 41.93 & 4.95 & 378 & 40.93 & 5.02 \\
\hline 8 & 401 & 240.51 & 53.58 & 405 & 250.31 & 58.60 & 402 & 44.04 & 5.41 & 405 & 43.02 & 5.59 \\
\hline 9 & 396 & 247.88 & 58.34 & 371 & 261.05 & 64.72 & 386 & 46.33 & 5.14 & 369 & 45.15 & 5.23 \\
\hline $10 \sim$ & 426 & 256.03 & 61.68 & 390 & 283.31 & 61.18 & 422 & 48.99 & 5.85 & 391 & 45.36 & 5.19 \\
\hline $11 \sim$ & 298 & 283.69 & 75.29 & 287 & 272.87 & 68.94 & 299 & 49.87 & 6.12 & 293 & 45.75 & 6.25 \\
\hline $12 \sim$ & 408 & 325.02 & 92.31 & 356 & 226.99 & 83.11 & 410 & 55.62 & 8.52 & 353 & 51.64 & 7.93 \\
\hline $13 \sim$ & 283 & 319.35 & 97.79 & 315 & 178.50 & 68.13 & 282 & 59.66 & 9.65 & 319 & 53.46 & 8.10 \\
\hline $14 \sim$ & 230 & 266.79 & 99.06 & 239 & 137.99 & 49.86 & 232 & 66.92 & 11.03 & 240 & 55.65 & 7.73 \\
\hline $15 \sim$ & 309 & 178.57 & 80.91 & 383 & 100.32 & 33.80 & 307 & 75.27 & 10.84 & 375 & 60.77 & 7.39 \\
\hline $16 \sim$ & 196 & 132.40 & 49.03 & 244 & 87.52 & 24.61 & 198 & 77.84 & 11.09 & 247 & 61.43 & 8.25 \\
\hline $17 \sim$ & 162 & 103.91 & 28.26 & 264 & 74.55 & 18.27 & 164 & 79.58 & 10.25 & 264 & 61.55 & 7.87 \\
\hline $18 \sim$ & 113 & 91.61 & 18.18 & 293 & 70.80 & 15.47 & 115 & 78.51 & 9.76 & 297 & 60.16 & 7.92 \\
\hline 19-20 & 63 & 83.44 & 19.79 & 168 & 69.28 & 14.40 & 63 & 84.51 & 8.72 & 165 & 59.67 & 6.85 \\
\hline
\end{tabular}


The results demonstrated that girls were more concentrated in the adolescent groups than boys (Fig. 2). Additionally, significant age dependence was shown in the trends of ALP and $\mathrm{Cr}$, and the results differed between the two analytes. For example, $\mathrm{Cr}$ continuously increased with age from 1 to 19 years, whereas a sharp decrease in ALP was observed after puberty (age 12 and 14 years for girls and boys, respectively). Cr showed the same tendency between boys and girls throughout the childhood phase, where boys plateau later than girls after a long period of growth. However, boys' and girls' ALP levels showed a decreasing trend in the first 4 years of life, but the levels then increased until puberty.

\section{Simulation of time frames for the improved non- parametric method}

The balance between the sample size in each time frame and the number of time frames was difficult to maintain. Through a process of statistical simulation, we obtained a figure with changing values of $n$ and $m(n=$ sample size in time frame, $m=$ mean difference between adjacent time frames), shown in Fig. 3. Although the curves obtained under various parameters were similar, we found that when the sample size is small (e.g., $n<$ 60 ), there were more discrete reference limit values, which may drift, influencing the curve fitting results. Moreover, when the sample size was too large (e.g. $n>$ 300 ), some details of the curve were lost, especially for ALP at ages 14-16 years, which showed a cliff-like descent. The Clinical and Laboratory Standards Institute (CLSI) recommends a minimum sample size of 120 to establish RIs. Combining CLSI's suggestion with Pavlov's research [16], we finally set the sample size within each time frame to 120 . Using an excessive number of time frames increases the arithmetic load. Therefore, the $n$ and $\mathrm{m}$ parameters were set as 120 and 2, respectively, for both ALP and Cr.

\section{Simulation of smoothing methods for the improved non- parametric method}

The AIC values for three smoothing methods are shown in Supplemental Table 1. Smoothing parameters were
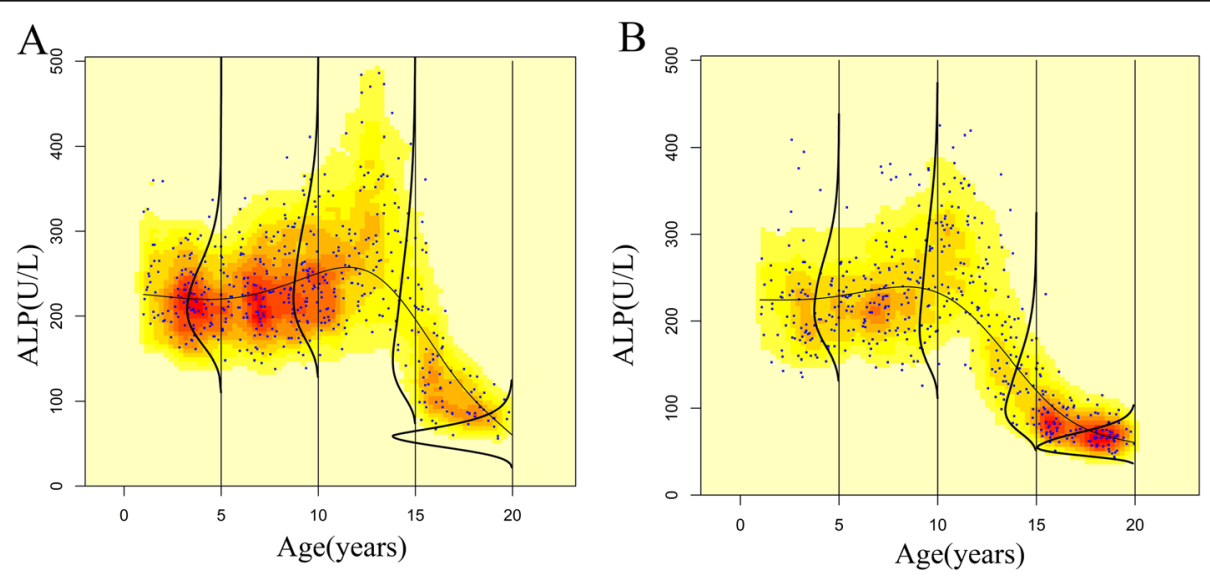

$\mathrm{C}$

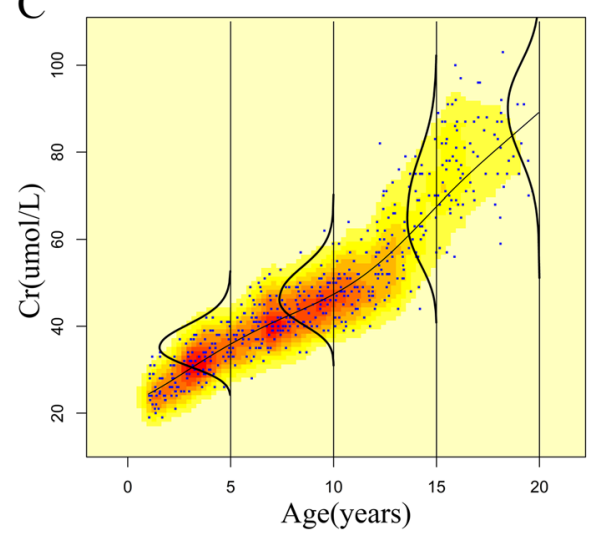

$\mathrm{D}$

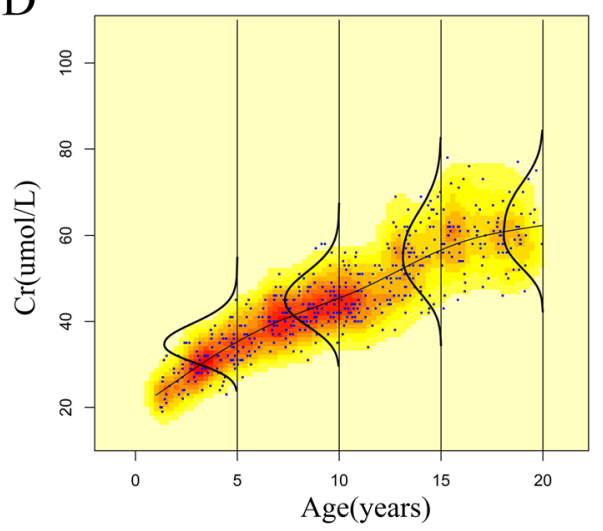

Fig. 2 The age dependent trend of alkaline phosphatase and serum creatinine by sex. a. alkaline phosphatase of boys. b. alkaline phosphatase of girls. c. serum creatinine of boys. d. serum creatinine of girls. ALP, alkaline phosphatase; Cr, serum creatinine. Notes: The center lines are fitted by GAMLSS method, the other curves are probability density functions and the horizontal axis represents the probability density for each age group. The density of the data points is represented by the color chromaticity 


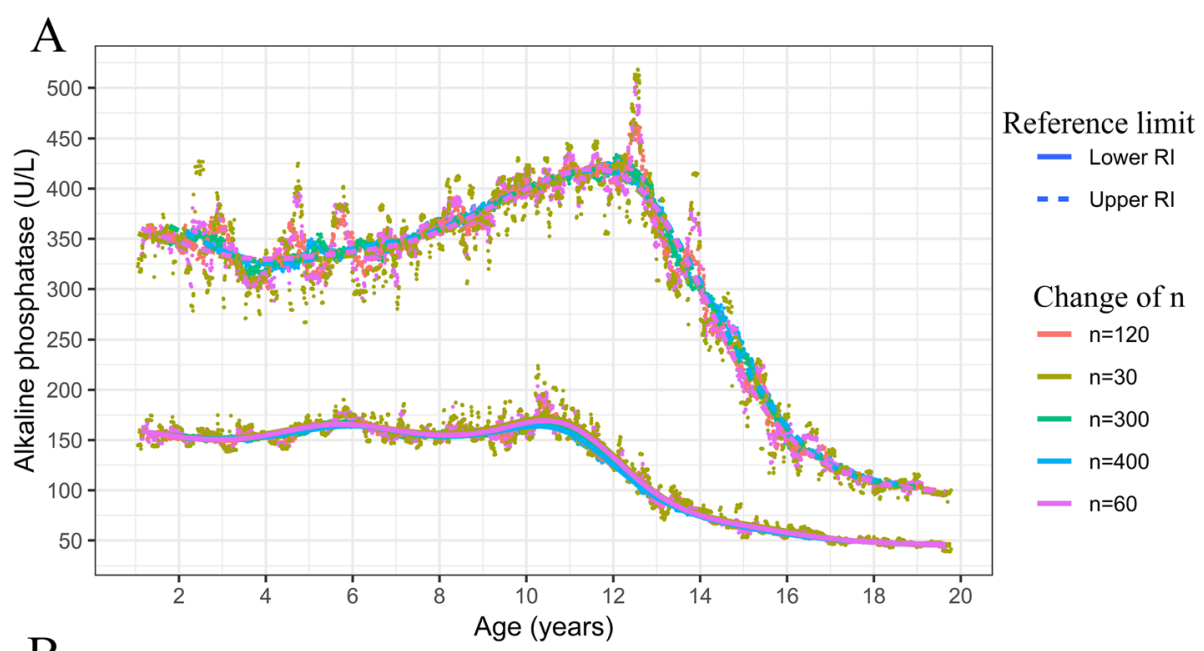

B

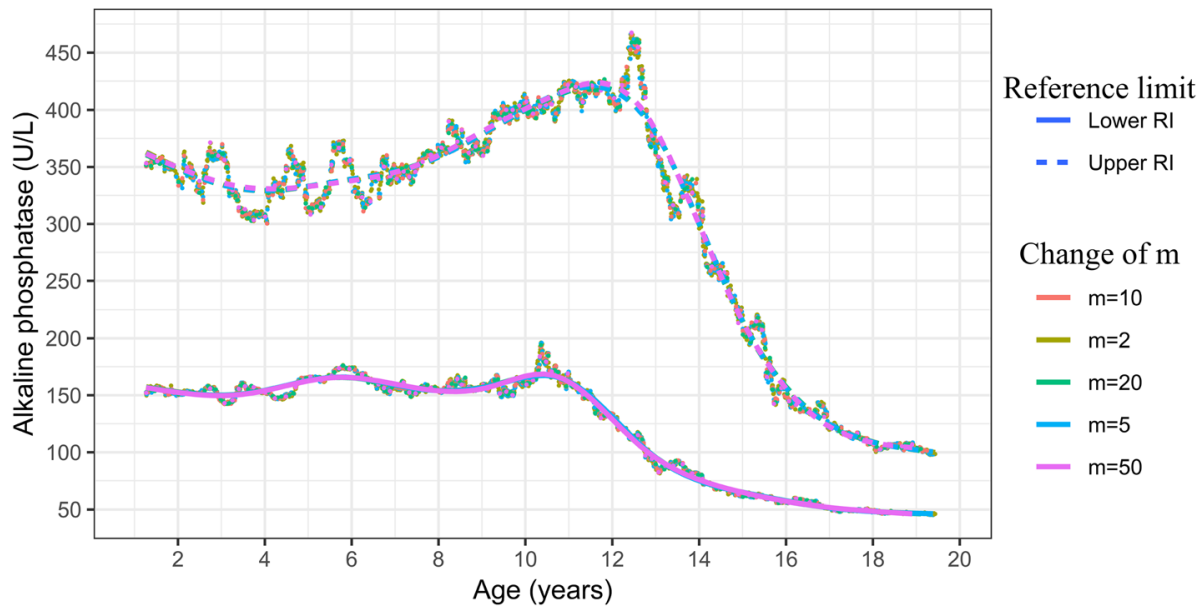

Fig. 3 Simulation of time frames with different sample sizes ( $n$ ) and mean difference between adjacent time frames ( $m$ ). a. Simulation of time frames with different sample sizes $(n), n=$ sample size in time frame. $\mathbf{b}$. Simulation of mean difference between adjacent time frames, $m=$ mean difference between adjacent time frames. ALP, alkaline phosphatase. Notes: Lines are fitted by cubic splines

selected by internal (i.e., local) maximum likelihood estimation in the $\mathrm{R}$ software package [24]. Among all models, the penalized spline method had the smallest AIC value. The continuous RIs fitted by penalized spline, cubic spline, and fraction polynomials are shown in Supplemental Figure 4. The fraction polynomials did not fit well at the end of the curve for ALP, and there was a cross between the upper and lower percentile curves. Furthermore, fluctuation occurred in the smooth curve simulated by the penalized spline method. Therefore, we adjusted the smoothing parameters of the cubic spline and penalized spline methods through visual inspection.

\section{Simulations based on GAMLSS and LMS}

Using the GAMLSS method, the four models were simulated using two distribution types (Box-Cox $t$ and BoxCox power exponential) and two smoothing methods (cubic spline or penalized spline). GAMLSS models' AIC values are shown in Supplemental Table 2. Compared with the cubic spline smoothing technique, penalized spline fit the data better according to the AIC value, similar to the simulation results of the non-parametric methods. The worm plots of simulations based on the LMS and GAMLSS methods demonstrate that the GAMLSS models fit the data better than LMS, especially for ALP (Supplemental Figure 5). This is because the GAMLSS model is more consistent with the theoretical distribution, in which data points are uniformly distributed on both sides of the center line [22].

\section{Continuous Rls for pediatric ALP and $\mathrm{Cr}$}

The continuous RIs were estimated using the improved non-parametric method, the RS method, the GAMLSS method, and the LMS method. Figure 4 shows the results of continuous RI estimation for $\mathrm{Cr}$ and ALP using the four methods. The RIs estimated by the LMS, 


\section{A Alkaline phosphatase of boys}

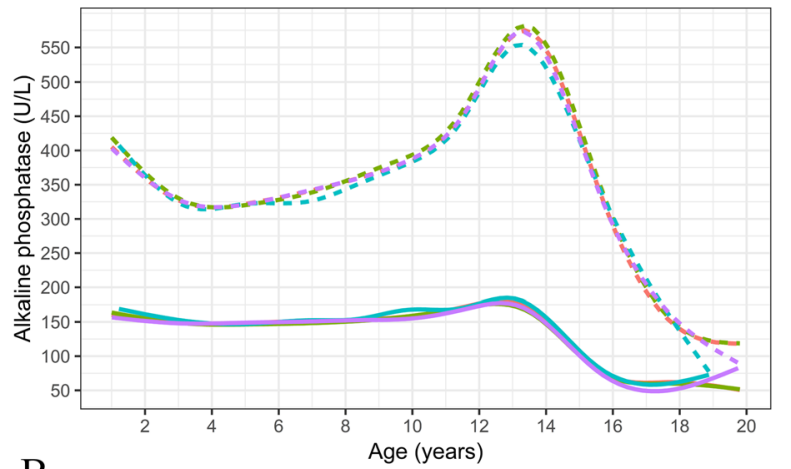

$$
\begin{aligned}
& \text { Method } \\
&- \text { GAMLSS } \\
&-\quad \text { LMS } \\
& \text { - Non-parametric }
\end{aligned}
$$

- RS

Percentile

- P2.5

- - P97.5

B

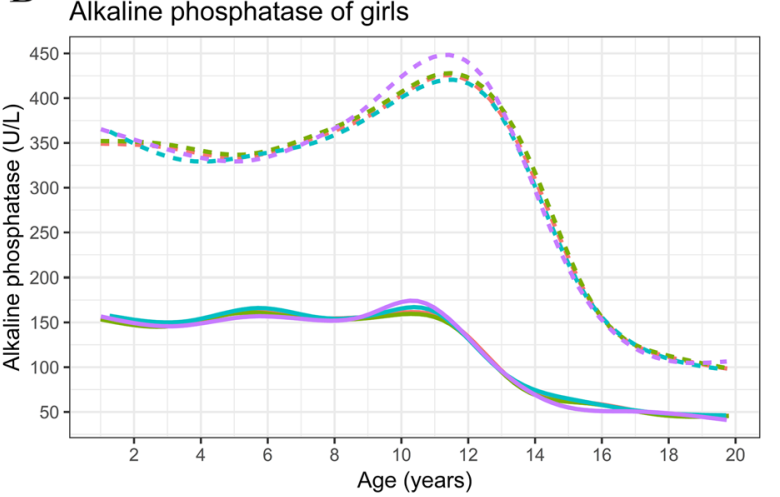

$$
\begin{aligned}
& \text { Method } \\
& \text { - } \text { GAMLSS } \\
& \text { - } \text { LMS } \\
& \text { — Non-parametric }
\end{aligned}
$$

- $\mathrm{RS}$

C serum creatinine of boys

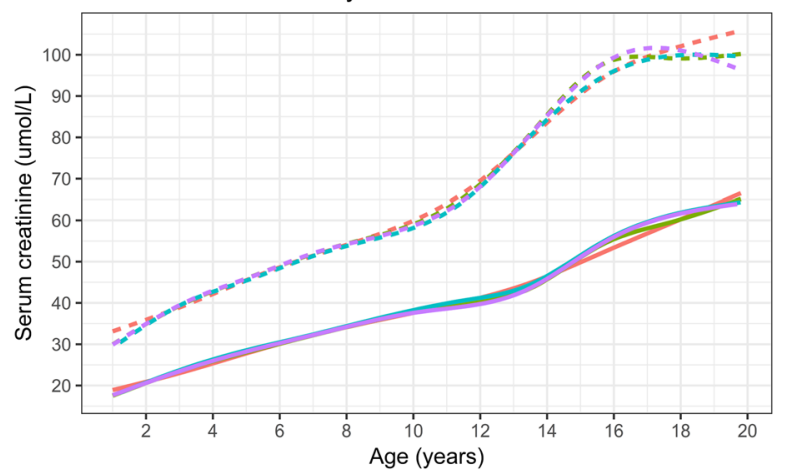

$$
\begin{array}{cl} 
& \text { Method } \\
\text { - } & \text { GAMLSS } \\
\text { - } & \text { LMS } \\
\text { — Non-parametric } & \text { Nons }
\end{array}
$$

- RS

\section{Percentile}

- P2.5

= - P97.5

\section{D serum creatinine of girls}

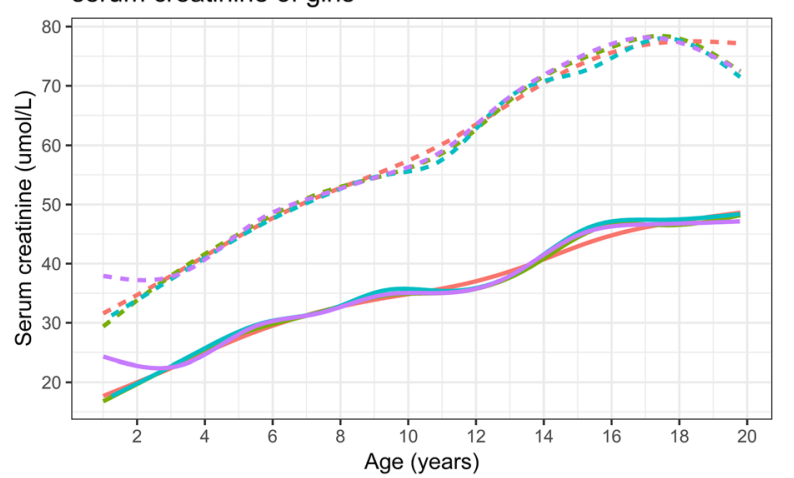

Method

- GAMLSS

- LMS

— Non-parametric

- RS

Percentile

- P2.5

- $\quad$ P97.5

Fig. 4 Continuous Rls of serum creatinine and alkaline phosphatase by sex. a. alkaline phosphatase of boys. $\mathbf{b}$. alkaline phosphatase of girls. c. serum creatinine of boys. $\mathbf{d}$. serum creatinine of girls. GAMLSS, General Additive Model for Location Scale and Shape method; LMS, LambdaMedian-Sigma method; RS, radial smoothing method; ALP, alkaline phosphatase; Cr, serum creatinine 
GAMLSS, and RS methods approximately overlapped, while the improved non-parametric method seemed better after visual inspection of the smoothing parameters. However, there were slight differences at the ends and peaks of the curves. Large edge effects were found in the curves fit by the RS method: left and right edge effects appeared for $\mathrm{Cr}$ and ALP, respectively. Age-specific reference values found for ALP and Cr using the GAMLSS method are presented in Tables 2 and 3.

Figure 5 shows the differences between discrete RIs partitioned by the decision tree technique and continuous RIs calculated by the GAMLSS method. The discrete RIs presented a ladder shape that jumped several times with increasing age. In addition, we added the continuous RIs from the CALIPER study [25]. The curves from CALIPER were smoother than this study's GAMLSS method results, especially for ALP in boys. Further, both the upper and lower reference limits of $\mathrm{Cr}$ calculated by CALIPER were slightly lower than those in the present study.

\section{Verifying Rls by test set}

The continuous RIs were verified using the test dataset. All OOR values in Table 4 were smaller than $10 \%$. The OOR percentages of the LMS, GAMLSS, and RS methods were much closer to $5 \%$, and both the lower OOR and upper OOR proportions were both close to $2.5 \%$. We also verified the continuous RIs calculated by CALIPER, all OOR values were less than $10 \%$, except for ALP of girls. In addition, we calculated the OOR rates of discrete RIs, which were also close to $5 \%$. Moreover, we created a table of OOR values for each year of age to ensure that the RIs accurately represent the relationship between age and analyte concentration (Supplemental Tables 3 and 4), which clearly showed the differences between continuous and discrete RIs. Although this study's sample size may be relatively insufficient, the OOR values of discrete RIs have larger variation in each age group compared with those of continuous RIs, especially near the thresholds of age divisions.

\section{Discussion}

Age partitioning is a common issue not only for pediatric RIs but also for other clinical laboratory indexes $[2,3,26,27]$. However, the use of age portioning methods to establish RIs still has some limitations, as the use of discrete age groups does not sensitively reflect continuous changes in growth and development. This problem is illustrated in Fig. 5. In contrast to the discrete RIs, the continuous RIs allow a precise

Table 2 Age-specific reference values for alkaline phosphatase

\begin{tabular}{|c|c|c|c|c|c|c|c|c|c|c|c|c|c|c|}
\hline \multirow[b]{3}{*}{ Age (years) } & \multicolumn{13}{|c|}{ Percentiles } & \multirow[b]{3}{*}{ 97.5th } \\
\hline & \multicolumn{7}{|c|}{ Alkaline phosphatase of boys (U/L) } & \multicolumn{6}{|c|}{ Alkaline phosphatase of girls $(\mathrm{U} / \mathrm{L})$} & \\
\hline & 2.5 th & 10th & 25 th & 50th & 75th & 90th & 97.5 th & 2.5 th & 10th & 25 th & 50th & 75th & 90th & \\
\hline$>=1$ & $158.12^{\mathrm{a}}$ & 183.98 & 211.87 & 249.58 & 295.47 & 344.57 & 410.22 & 147.65 & 170.83 & 194.89 & 226.17 & 260.05 & 290.23 & 323.58 \\
\hline $2 \sim$ & 154.04 & 176.04 & 199.47 & 230.77 & 267.96 & 306.45 & 356.10 & 150.59 & 172.39 & 196.48 & 230.20 & 270.07 & 308.80 & 355.40 \\
\hline $3 \sim$ & 149.78 & 169.74 & 190.93 & 219.21 & 252.45 & 286.19 & 328.80 & 149.71 & 169.01 & 190.69 & 221.77 & 259.78 & 298.16 & 346.36 \\
\hline 4 & 145.69 & 164.91 & 185.44 & 213.02 & 245.43 & 277.99 & 318.70 & 149.40 & 166.49 & 185.77 & 213.65 & 248.26 & 283.90 & 329.80 \\
\hline 5 & 146.41 & 165.46 & 185.90 & 213.56 & 246.01 & 278.29 & 318.22 & 159.86 & 176.36 & 195.03 & 222.15 & 256.15 & 291.68 & 338.35 \\
\hline 6 & 154.68 & 174.62 & 196.14 & 225.44 & 259.80 & 293.67 & 335.16 & 165.39 & 182.25 & 201.18 & 228.42 & 262.13 & 296.88 & 341.79 \\
\hline 7 & 149.28 & 169.46 & 191.51 & 221.98 & 258.00 & 293.50 & 336.94 & 156.96 & 175.67 & 196.59 & 226.41 & 262.79 & 299.55 & 345.84 \\
\hline 8 & 151.66 & 173.21 & 197.06 & 230.53 & 270.47 & 309.86 & 358.01 & 152.36 & 174.93 & 199.89 & 234.82 & 276.26 & 316.78 & 365.84 \\
\hline 9 & 156.19 & 178.24 & 202.77 & 237.41 & 278.74 & 319.15 & 368.11 & 153.30 & 180.38 & 209.44 & 248.57 & 292.80 & 333.93 & 381.25 \\
\hline $10 \sim$ & 159.57 & 182.49 & 208.21 & 244.90 & 288.83 & 331.60 & 383.14 & 163.50 & 194.82 & 227.33 & 269.50 & 315.23 & 356.14 & 401.53 \\
\hline $11 \sim$ & 163.55 & 188.80 & 217.60 & 259.44 & 310.24 & 359.94 & 420.05 & 163.59 & 197.41 & 232.63 & 278.44 & 328.23 & 372.88 & 422.47 \\
\hline $12 \sim$ & 177.13 & 206.76 & 241.16 & 292.20 & 355.15 & 417.23 & 492.69 & 128.62 & 160.81 & 196.43 & 245.75 & 303.01 & 357.46 & 421.22 \\
\hline $13 \sim$ & 183.35 & 217.16 & 257.27 & 318.29 & 395.16 & 471.87 & 566.02 & 93.35 & 117.65 & 146.58 & 190.35 & 246.77 & 306.19 & 383.20 \\
\hline $14 \sim$ & 149.89 & 181.66 & 220.51 & 281.75 & 361.49 & 442.93 & 544.83 & 74.21 & 90.45 & 110.36 & 141.94 & 185.71 & 236.02 & 308.37 \\
\hline $15 \sim$ & 100.65 & 124.89 & 155.45 & 205.40 & 272.68 & 343.05 & 432.82 & 63.30 & 74.12 & 87.20 & 107.79 & 136.42 & 169.87 & 219.54 \\
\hline $16 \sim$ & 71.07 & 87.33 & 107.74 & 140.90 & 184.96 & 230.14 & 286.59 & 55.64 & 63.43 & 72.59 & 86.56 & 105.25 & 126.27 & 156.26 \\
\hline $17 \sim$ & 62.80 & 73.95 & 87.40 & 108.24 & 134.33 & 159.48 & 189.13 & 50.72 & 57.23 & 64.69 & 75.68 & 89.73 & 104.74 & 124.89 \\
\hline 18 & 59.95 & 68.26 & 77.98 & 92.52 & 109.90 & 125.85 & 143.82 & 47.53 & 53.67 & 60.54 & 70.35 & 82.37 & 94.60 & 110.11 \\
\hline 19 & 56.06 & 62.90 & 70.82 & 82.53 & 96.23 & 108.52 & 122.04 & 45.38 & 51.64 & 58.49 & 67.96 & 79.08 & 89.88 & 102.90 \\
\hline
\end{tabular}

${ }^{a}$ Calculated by GAMLSS method 
Table 3 Age-specific reference values for creatinine

\begin{tabular}{|c|c|c|c|c|c|c|c|c|c|c|c|c|c|c|}
\hline \multirow[b]{3}{*}{ Age (years) } & \multicolumn{13}{|c|}{ Percentiles } & \multirow[b]{3}{*}{97.5 th } \\
\hline & \multicolumn{7}{|c|}{ Serum creatinine of boys (umol/L) } & \multicolumn{6}{|c|}{ Serum creatinine of girls (umol/L) } & \\
\hline & 2.5 th & 10th & 25 th & 50th & 75th & 90th & 97.5 th & 2.5th & 10th & 25 th & 50th & 75th & 90th & \\
\hline$>=1$ & $19.01^{\mathrm{a}}$ & 21.11 & 23.18 & 25.72 & 28.37 & 30.71 & 33.28 & 17.75 & 19.83 & 21.78 & 24.03 & 26.43 & 28.79 & 31.65 \\
\hline $2 \sim$ & 20.84 & 22.97 & 25.09 & 27.71 & 30.49 & 33.02 & 35.85 & 19.96 & 22.13 & 24.18 & 26.57 & 29.13 & 31.63 & 34.67 \\
\hline 3 & 23.01 & 25.20 & 27.38 & 30.09 & 33.03 & 35.76 & 38.92 & 22.42 & 24.66 & 26.81 & 29.35 & 32.09 & 34.74 & 37.93 \\
\hline $4 \sim$ & 25.38 & 27.64 & 29.90 & 32.72 & 35.81 & 38.75 & 42.22 & 24.94 & 27.26 & 29.52 & 32.23 & 35.16 & 37.96 & 41.28 \\
\hline $5 \sim$ & 27.77 & 30.13 & 32.49 & 35.43 & 38.67 & 41.78 & 45.48 & 27.36 & 29.78 & 32.15 & 35.05 & 38.18 & 41.13 & 44.60 \\
\hline 6 & 30.07 & 32.54 & 35.02 & 38.10 & 41.48 & 44.72 & 48.59 & 29.51 & 32.07 & 34.58 & 37.66 & 40.97 & 44.08 & 47.70 \\
\hline 7 & 32.20 & 34.80 & 37.38 & 40.60 & 44.13 & 47.48 & 51.44 & 31.29 & 34.02 & 36.69 & 39.94 & 43.42 & 46.66 & 50.42 \\
\hline $8 \sim$ & 34.14 & 36.84 & 39.54 & 42.91 & 46.59 & 50.04 & 54.06 & 32.72 & 35.64 & 38.48 & 41.91 & 45.55 & 48.92 & 52.80 \\
\hline 9 & 35.91 & 38.72 & 41.56 & 45.14 & 49.03 & 52.62 & 56.73 & 33.89 & 37.03 & 40.06 & 43.70 & 47.53 & 51.04 & 55.04 \\
\hline $10 \sim$ & 37.60 & 40.55 & 43.58 & 47.50 & 51.75 & 55.59 & 59.89 & 34.85 & 38.27 & 41.55 & 45.47 & 49.55 & 53.24 & 57.39 \\
\hline $11 \sim$ & 39.30 & 42.47 & 45.81 & 50.23 & 55.07 & 59.33 & 64.01 & 35.78 & 39.53 & 43.12 & 47.41 & 51.83 & 55.78 & 60.15 \\
\hline $12 \sim$ & 41.24 & 44.75 & 48.52 & 53.63 & 59.25 & 64.16 & 69.50 & 37.00 & 41.08 & 45.00 & 49.68 & 54.50 & 58.77 & 63.47 \\
\hline 13 & 43.58 & 47.59 & 51.92 & 57.83 & 64.35 & 70.07 & 76.27 & 38.69 & 43.00 & 47.16 & 52.20 & 57.41 & 62.00 & 67.04 \\
\hline $14 \sim$ & 46.41 & 51.07 & 56.04 & 62.73 & 70.06 & 76.52 & 83.56 & 40.74 & 45.14 & 49.45 & 54.73 & 60.24 & 65.10 & 70.43 \\
\hline 15 & 49.77 & 55.12 & 60.68 & 67.96 & 75.84 & 82.79 & 90.42 & 42.89 & 47.27 & 51.63 & 57.06 & 62.77 & 67.82 & 73.38 \\
\hline $16 \sim$ & 53.41 & 59.41 & 65.43 & 72.98 & 81.00 & 88.11 & 95.95 & 44.78 & 49.09 & 53.43 & 58.90 & 64.72 & 69.90 & 75.64 \\
\hline $17 \sim$ & 56.89 & 63.51 & 69.84 & 77.38 & 85.14 & 92.02 & 99.62 & 46.17 & 50.41 & 54.69 & 60.11 & 65.91 & 71.14 & 76.98 \\
\hline $18 \sim$ & 60.21 & 67.38 & 73.88 & 81.22 & 88.53 & 94.97 & 102.02 & 47.15 & 51.31 & 55.50 & 60.79 & 66.48 & 71.64 & 77.46 \\
\hline $19-20$ & 63.66 & 71.26 & 77.81 & 84.86 & 91.68 & 97.65 & 104.14 & 47.99 & 52.01 & 56.07 & 61.19 & 66.72 & 71.73 & 77.40 \\
\hline
\end{tabular}

${ }^{a}$ Calculated by GAMLSS method

representation of age and sex-dependent change during growth and development. Therefore, to provide evidence for the applicability of different algorithms to establish continuous RIs, we presented continuous RIs simulated by four methods from infancy to adulthood.

The age-dependent trends of ALP and $\mathrm{Cr}$ in the present study were consistent with those in previous studies, which represent distinctive age-dependent trends $[6,25]$. Different from study reported by Zierk [6], in which data were collected from hospitals, all the reference individuals were healthy children recruited for the PRINCE study. Moreover, there were slight differences in the continuous RIs between the CALIPER study and the present study: the reference limits were slightly higher in the present study, which may be caused by differences in the reference population and inspection instruments.

Establishing a reference interval using a nonparametric method is an indirect process: curve fitting is simulated considering only the values of discrete reference limits, rather than including all data. This weakness leads to curve fluctuations, even if it has the best AIC value and appropriate smoothing parameters. Our research indicates that although the model can obtain a better AIC value, the smoothing parameters adjusted by visual inspection better represent the whole dataset and are more suitable in the non-parametric methods. Therefore, the trends of age dependence should be fully understood before establishing continuous RIs using the non-parametric methods. Moreover, it is necessary to adjust the smoothing parameters through visual inspection instead of only relying on software algorithms.

We used a robust bootstrap method to estimate the discrete RIs for each time frame in the improved nonparametric method, after considering the accuracy and feasibility of various methods. In our study, not all analyte levels had normal distributions across the 200,000 time frames. Therefore, the data should be transformed to a normal distribution if we use parametric methods to calculate RIs. However, hypotheses testing and data transformation for large datasets depend on programming capabilities and statistical package functions. We tried to use the powertransform function in $\mathrm{R}$ to perform the Box-Cox transformation, but there were still some time frames for which the best $\lambda$ could not be obtained, and those needed to be debugged manually. However, manual debugging would incur an inestimable time cost. According Pavlov's research [16], the bootstrap method has relatively high accuracy when the sample size is relatively small, so we chose the bootstrap method for the improved non-parametric procedure. 


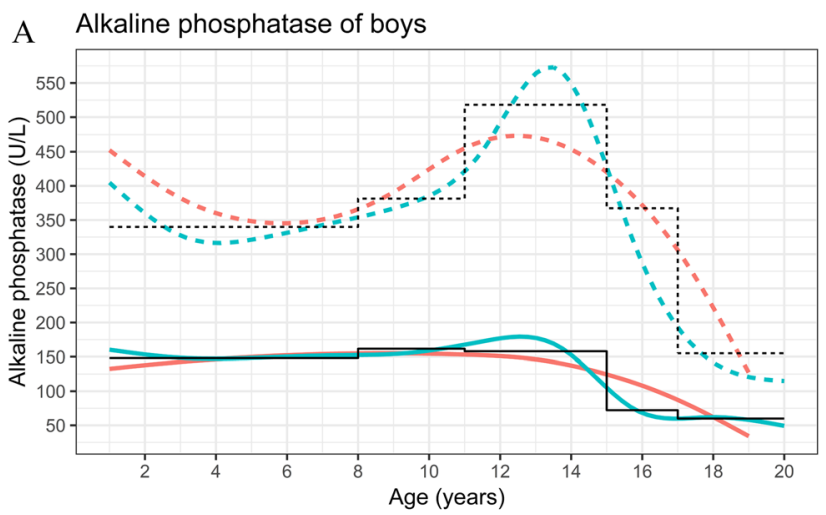

$$
\begin{aligned}
& \text { Method } \\
& \text { - CALIPER } \\
& \text { — GAMLSS } \\
& \text { —-Discrete RIs } \\
& \text { Percentile } \\
& \text { - P2.5 } \\
& \text { - - P97.5 }
\end{aligned}
$$

B Alkaline phosphatase of girls

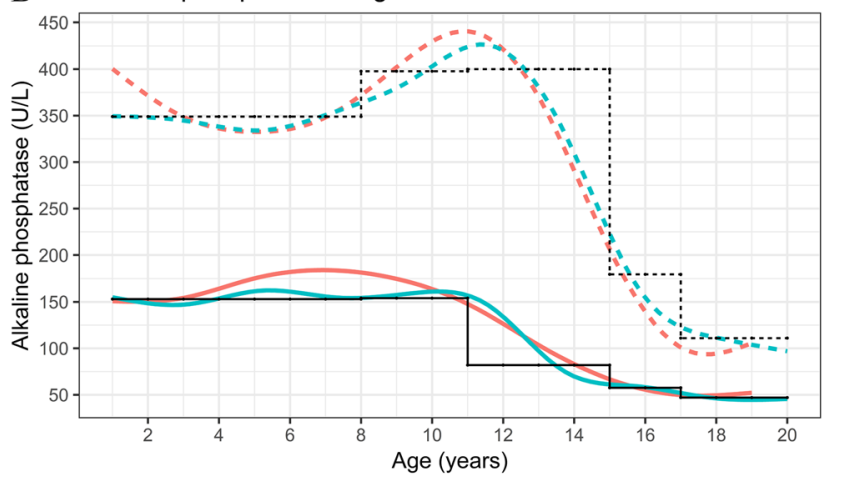

Method

- CALIPER

- GAMLSS

—Discrete RIs

Percentile

- P2.5

- - P97.5

$\mathrm{C}_{110}$ serum creatinine of boys

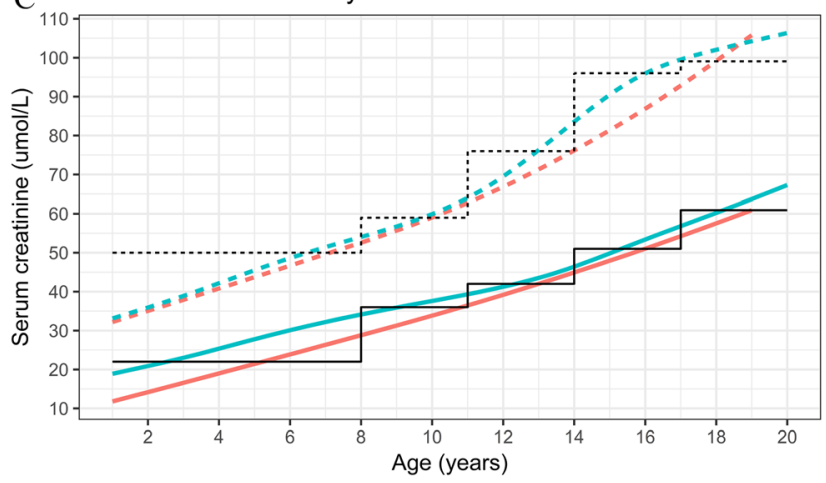

Method

- CALIPER

- GAMLSS

—Discrete RIs

Percentile

- P2.5

- - P97.5

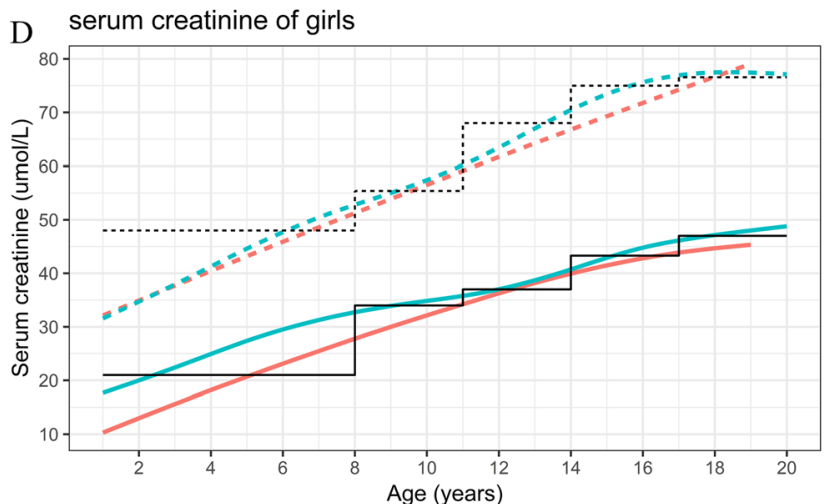

Metho

- CALIPER

— GAMLSS

—Discrete RIs

Percentile

- P2.5

- - P97.5

Fig. 5 Comparing the RIs with CALIPER study. a. alkaline phosphatase of boys. b. alkaline phosphatase of girls. c. serum creatinine of boys. d. serum creatinine of girls. ALP, alkaline phosphatase; Cr, serum creatinine; GAMLSS, General Additive Model for Location Scale and Shape method; CALIPER: Canadian Laboratory Initiative in Pediatric Reference Intervals 
Table 4 Out of range (OOR) of different simulation methods verified with test data set

\begin{tabular}{|c|c|c|c|}
\hline Model & Total of OOR (\%) & Lower OOR (\%) & Upper OOR (\%) \\
\hline \multicolumn{4}{|l|}{ Serum creatinine of boys } \\
\hline Improved non-parametric ${ }^{a}$ & $6.50^{b}$ & 3.34 & 3.16 \\
\hline RS & 4.87 & 2.39 & 2.47 \\
\hline LMS & 5.18 & 2.62 & 2.57 \\
\hline GAMLSS $^{\mathrm{C}}$ & 5.03 & 2.38 & 2.65 \\
\hline Discrete reference interval & 4.32 & 2.08 & 2.25 \\
\hline CALIPER & 7.58 & 0.57 & 7.01 \\
\hline \multicolumn{4}{|l|}{ Serum creatinine of girls } \\
\hline Improved Non-parametric & 6.62 & 3.34 & 3.28 \\
\hline RS & 5.21 & 2.60 & 2.62 \\
\hline LMS & 4.94 & 2.48 & 2.46 \\
\hline GAMLSS & 5.45 & 2.85 & 2.60 \\
\hline Discrete reference interval & 4.17 & 2.00 & 2.18 \\
\hline CALIPER & 6.13 & 0.84 & 5.28 \\
\hline \multicolumn{4}{|l|}{ Alkaline phosphatase of boys } \\
\hline Improved non-parametric & 7.01 & 3.69 & 3.31 \\
\hline RS & 5.26 & 2.75 & 2.50 \\
\hline LMS & 4.74 & 2.46 & 2.29 \\
\hline GAMLSS & 5.04 & 2.51 & 2.53 \\
\hline Discrete reference interval & 5.66 & 3.03 & 2.63 \\
\hline CALIPER & 6.81 & 4.41 & 2.39 \\
\hline \multicolumn{4}{|l|}{ Alkaline phosphatase of girls } \\
\hline Improved non-parametric & 6.72 & 3.59 & 3.13 \\
\hline RS & 4.53 & 2.30 & 2.23 \\
\hline LMS & 4.94 & 2.48 & 2.46 \\
\hline GAMLSS & 5.02 & 2.43 & 2.59 \\
\hline Discrete reference interval & 4.90 & 2.32 & 2.59 \\
\hline CALIPER & 10.22 & 7.10 & 3.12 \\
\hline
\end{tabular}

OOR Out of range, GAMLSS General Additive Model for Location Scale and Shape method, CALIPER Canadian Laboratory Initiative in Pediatric Reference Intervals, LMS Lambda-Median-Sigma method, RS radial smoothing method

a Smoothing parameters are selected by visual inspection of non-parametric method

${ }^{b}$ All values are calculated as mean of the results of 100 times simulation

c The models of GAMLSS method are selected from Table 3, which AIC is smallest

Additionally, LMS and GAMLSS have been widely applied to establish growth curves. They also perform well at establishing continuous RIs for analytes. As presented, the OOR percentages of those two methods were close to $5 \%$. In contrast, the OOR proportions of the nonparametric methods were more than $6 \%$ for ALP, which means that the non-parametric methods' RIs are narrower than those of the LMS and GAMLSS methods. Further, both the GAMLSS and LMS methods are simple to implement and adapt to complex age-dependent trends, especially when the age distribution of the analyte's concentration is not fully understood.

As a new approach for estimation of age-specific reference percentile curves, the RS method performs well at growth curve establishment [8]. However, it did not generate effective RIs for ALP without data transformation by the Box-Cox method (Supplemental Figure 6). This means that the distribution of data should be approximately normal, especially when the analyte has a more intricate age-dependent trend.

As for verification results, all four methods' OOR values were less than $10 \%$, which means that all methods showed good fit for establishing continuous RIs of $\mathrm{Cr}$ and ALP. However, edge effects were observed in all of the curves fit by these four methods. Even if the smoothing parameters were adjusted by visual inspection, the drift at the end of the curve was still not improved in the non-parametric methods. This phenomenon was 
most prominent when the RS method was used. These results could be attributed to the limited sample size of reference individuals. In our study, the number of reference individuals in the 19-year-old age group of boys was less than 100 for both ALP and Cr, which is insufficient compared with the other age groups. In contrast, the WHO Multicenter Growth Reference Study enlarged the birth sample to 1737 to minimize the left edge effect [7]. It is particularly difficult to sample more reference individuals aged less than 1 year. Although we removed the reference individuals aged less than 1 year to lessen the edge effects, the sample size of infants is not sufficient. Therefore, a larger sample size would be needed to establish continuous RIs. In comparison with the non-parametric methods, the LMS and GAMLSS methods have fewer edge effects when sample size is relatively lacking. In addition, LMS and GAMLSS are easy to implement and have high accuracy, which could be factors to recommend them as convenient and accurate methods for clinical establishment of RIs.

Other factors besides age and sex, such as height and weight, may also affect analyte levels. In future research to establish RIs, multifactorial analysis could be considered. Further, the opinions of clinicians and laboratory physicians should be taken into consideration during the variable selection process. All of these directions would ultimately lead to huge challenges in terms of model selection and subject recruitment.

Moreover, different methods often estimated the upper and lower limits with the least amount of bias [1]. The idea of establishing reference limits with two different methods was previously explored by Horn PS et al. [28].
There is a huge gap between the establishment of RIs and clinical practice. A possible solution is to integrate continuous RIs into laboratory testing platforms. The obtained models could be embedded into hospital clinical laboratory testing systems, and the RIs could be obtained from the models according to the information needed. Other quantile curves, such as the 5th, 25th, 50th, and 75th percentiles, can be easily obtained from the model. Therefore, doctors could not only judge whether the individual's laboratory result is abnormal but also provide a graph to present the patient's level compared with continuous RIs. In addition, longitudinal dynamic trends can be determined when individuals have multi-time laboratory results within a certain period (Fig. 6). Compared with a single test, the dynamic trends of some analytes could provide more diagnostic information about changes to individual health status. Moreover, graphical displays of clinical laboratory analytes would provide an improvement in clinical laboratory reporting.

The concept of continuous RIs is timeless and should become a standard throughout the entire field of laboratory medicine. It is necessary to establish continuous RIs for all ages rather than only focusing on the initial stages of life. When we are limited to the reference population, we cannot make such age divisions. Mørkrid et al. presented an elegant example of this viewpoint [29].

\section{Conclusions}

Four statistical methods to estimate continuous RIs for ALP and $\mathrm{Cr}$ were simulated and verified. The verification of continuous RIs showed that all four methods could be used to establish continuous RIs of clinical laboratory analytes. The GAMLSS and LMS methods were
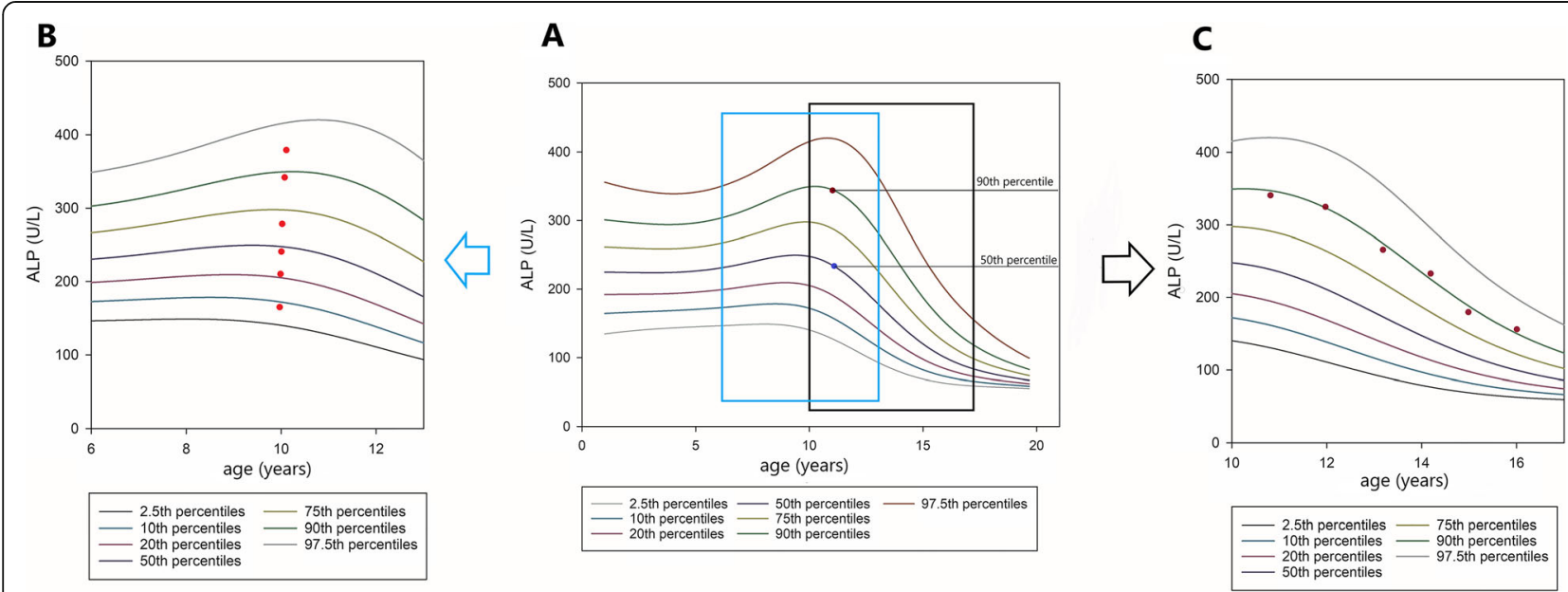

Fig. 6 The application of continuous reference intervals. The seven curves denote the 97.5th, 90th, 75th, 50th, 20th, 10th and 2.5th percentiles for ALP of girls respectively. Dots show the result of individual laboratory examination. $\mathbf{a}$. The percentile curves for ALP of boys aged 1 to 19 years. $\mathbf{b}$. During hospitalization, patient's ALP continued to increase for several times. c. With the increase of age, patient's ALP decreased. ALP, alkaline phosphatase 
more reliable than the RS and non-parametric methods, especially when sample size was insufficient. Therefore, the former two can be recommended as convenient and accurate methods for RIs establishment in clinical practice. In addition, the distribution of the data should be approximately normal when using the RS method to establish continuous RIs.

\section{Supplementary information}

Supplementary information accompanies this paper at https://doi.org/10. 1186/s12874-020-01021-y.

\section{Additional file 1.}

\section{Abbreviations}

ALP: Alkaline phosphatase; Cr: Serum creatinine; Rls: Reference intervals; OOR: Out of range; PRINCE: Pediatric Reference Interval in China study; CALIPER: Canadian Laboratory Initiative in Pediatric Reference Intervals; GAMLSS: The General Additive Model for Location Scale and Shape method; LMS: The Lambda-Median-Sigma method; RS: The radial smoothing method; $B C T$ : The Box-Cox t distribution; BCPE: The Box-Cox power exponential distribution

\section{Acknowledgments}

We acknowledge all participants for their cooperation and sample contributions. We also thank Richard Lipkin, PhD, from Liwen Bianji, Edanz Group China, for editing the English text of a draft of this manuscript.

\section{Authors' contributions}

PX and LK contributed to the study design. HL, SW and LQ contributed to the data acquisition. PY and YR provided statics guidance. LK contributed to drafting the manuscript. PX and NX contributed to revising the manuscript for important intellectual content. All authors read and approved the final manuscript.

\section{Funding}

Medical hospital authority, National Health Commission of the People's Republic of China (No. 2017374), only as a unique financial support for this project, has no interest and role in the design and performance, or in the data collection, analysis and interpretation of the results, or in the preparation and approval of this manuscript. This work was also supported by Beijing Municipal Administration of Hospitals Clinical medicine Development of Special Grant (No. ZY201404), Pediatric Medical Coordinated Development, Center of Beijing Municipal Administration of Hospitals (No. XTCX201812), and Capital Medical development research foundation (20192-2096).

\section{Availability of data and materials}

The data are not publicly available as they contain information that could compromise research participant privacy. But they are available from the corresponding author on reasonable request. The request to access the raw data treated by deidentification from the PRINCE study must be approved by Academic Committee of PRINCE study.

\section{Ethics approval and consent to participate}

The PRINCE study was approved by the Institutional Review Board of Beijing Children's Hospital (IEC-C-028-A10-V.05). At the same time, the protocol was approved by the institutional review boards of other 10 collaborating centers. Informed written consent was signed by the participant's legally authorized representative (the parent or guardian) in the case of young children aged less than 8 years. Otherwise, the informed consent should be signed by both children himself and the participant's legally authorized representative [10].

\section{Consent for publication}

Not applicable.

\section{Competing interests}

The authors declare that they have no competing interests.

\section{Author details}

'Department of Epidemiology and Health Statistics, School of Public Health, Capital Medical University, Beijing 100069, China. ${ }^{2}$ Center for Clinical Epidemiology and Evidence-based Medicine, Beijing Children's Hospital, Capital Medical University, National Center for Children Health, No.56 Nanlishi Road, Beijing 100045, China. ${ }^{3}$ Department of Clinical Laboratory Center, Beijing Children's Hospital, Capital Medical University, National Center for Children Health, No.56 Nanlishi Road, Beijing 100045, China. ${ }^{4}$ Beijing Key Laboratory for Pediatric Diseases of Otolaryngology, Head and Neck, Surgery, Beijing Children's Hospital, Capital Medical University, National Center for Children Health, No.56 Nanlishi Road, Beijing 100045, China.

Received: 20 August 2019 Accepted: 18 May 2020

Published online: 01 June 2020

\section{References}

1. Daly CH, Higgins V, Adeli K, Grey VL, Hamid JS. Reference interval estimation: methodological comparison using extensive simulations and empirical data. Clin Biochem. 2017;50(18):1145-58.

2. Peng $X, L v$ Y, Feng G, Peng Y, Li Q, Song W, Ni X. Algorithm on age partitioning for estimation of reference intervals using clinical laboratory database exemplified with plasma creatinine. Clin Chem Lab Med. 2018; 56(9):1514-23.

3. Zierk J, Arzideh F, Haeckel R, Rascher W, Rauh M, Metzler M. Indirect determination of pediatric blood count reference intervals. Clin Chem Lab Med. 2013;51(4):863-72.

4. CLSI. Establishing, and verifying reference intervals in the clinical laboratory; approved guideline - third edition. CLSI Document EP28-A3C 2008.

5. Zierk J, Arzideh F, Haeckel R, Cario H, Fruhwald MC, Gross HJ, Gscheidmeier $T$, Hoffmann R, Krebs A, Lichtinghagen R, et al. Pediatric reference intervals for alkaline phosphatase. Clin Chem Lab Med. 2017;55(1):102-10.

6. Zierk J, Arzideh F, Rechenauer T, Haeckel R, Rascher W, Metzler M, Rauh M. Age- and sex-specific dynamics in 22 hematologic and biochemical analytes from birth to adolescence. Clin Chem. 2015;61(7):964-73.

7. Borghi E, de Onis M, Garza C, Van den Broeck J, Frongillo EA, GrummerStrawn L, Van Buuren S, Pan H, Molinari L, Martorell R, et al. Construction of the World Health Organization child growth standards: selection of methods for attained growth curves. Stat Med. 2006;25(2):247-65.

8. Wan X, Qu Y, Huang Y, Zhang $X$, Song H, Jiang H. Nonparametric estimation of age-specific reference percentile curves with radial smoothing. Contemp Clin Trials. 2012;33(1):13-22.

9. De Henauw S, Michels N, Vyncke K, Hebestreit A, Russo P, Intemann T, Peplies J, Fraterman A, Eiben G, de Lorgeril M, et al. Blood lipids among young children in Europe: results from the European IDEFICS study. Int J Obes. 2014;38(Suppl 2):S67-75.

10. Ni X, Song W, Peng $X$, Shen Y, Peng Y, Li Q, Wang Y, Hu L, Cai Y, Shang H, et al. Pediatric reference intervals in China (PRINCE): design and rationale for a large, multicenter collaborative cross-sectional study. Sci Bull. 2018;63(24): 1626-34.

11. Stasinopoulos DM, Rigby RA. Generalized additive models for location scale and shape (GAMLSS) in R. J R Stat Soc. 2005;54(3):507-54.

12. Cole TJ. Using the LMS method to measure skewness in the NCHS and Dutch National height standards. Ann Hum Biol. 1989;16(5):407-19.

13. Cole TJ, Green PJ. Smoothing reference centile curves: the LMS method and penalized likelihood. Stat Med. 1992;11(10):1305-19.

14. Royston P, Altman DG. Regression using fractional polynomials of continuous covariates: parsimonious parametric modeling. J R Stat Soc. 1994;43(3):429-67.

15. Arzideh F, Wosniok W, Haeckel R. Indirect reference intervals of plasma and serum thyrotropin (TSH) concentrations from intra-laboratory data bases from several German and Italian medical centres. Clin Chem Lab Med. 2011; 49(4):659-64.

16. Pavlov IY, Wilson AR, Delgado JC. Reference interval computation: which method (not) to choose? Clin Chim Acta. 2012;413(13-14):1107-14.

17. Akaike H. A new look at the statistical model identification. IEEE Trans Automatic Control. 1974;19(6):716-23.

18. Hastie T, Tibshirani R. Generalized additive models. Stat Sci. 1986;1(3):297310. 
19. Lane PW, Wood S, Jones MC, Nelder JA, Lee YJ, Borja MC, Longford NT, Bowman A, Cole TJ. Generalized additive models for location, scale and shape - discussion. Appl Stat. 2005;54:544-54.

20. Rigby RA, Stasinopoulos DM. Using the box-cox t distribution in GAMLSS to model skewness and kurtosis. Stat Model. 2006;6(6):209-29.

21. Rigby RA, Stasinopoulos DM. Smooth centile curves for skew and kurtotic data modelled using the box-cox power exponential distribution. Stat Med. 2004;23(19):3053-76.

22. van Buuren S, Fredriks M. Worm plot: a simple diagnostic device for modelling growth reference curves. Stat Med. 2001;20(8):1259-77.

23. Jaschke SR: The cornish-fisher-expansion in the context of delta - gamma normal approximations. Sfb Discussion Papers 2001.

24. Rigby RA, Stasinopoulos DM. Automatic smoothing parameter selection in GAMLSS with an application to centile estimation. Stat Methods Med Res. 2014;23(4):318-32.

25. Asgari S, Higgins V, McCudden C, Adeli K. Continuous reference intervals for 38 biochemical markers in healthy children and adolescents: comparisons to traditionally partitioned reference intervals. Clin Biochem. 2019;73:82-9.

26. Schnabl K, Chan MK, Gong Y, Adeli K. Closing the gaps in paediatric reference intervals: the CALIPER initiative. Clin Biochem Rev. 2008;29(3): 89-96.

27. LV Y, Feng $G, N i X$, Song $W$, Peng $X$. The critical gap for pediatric reference intervals of complete blood count in China. Clin Chim Acta. 2017;469:22-5.

28. Horn PS, Pesce AJ, Copeland BE. A robust approach to reference interval estimation and evaluation. Clin Chem. 1998;44(3):622-31.

29. Morkrid L, Rowe AD, Elgstoen KB, Olesen $J H$, Ruijter G, Hall PL, Tortorelli S, Schulze A, Kyriakopoulou L, Wamelink MM, et al. Continuous age- and sexadjusted reference intervals of urinary markers for cerebral creatine deficiency syndromes: a novel approach to the definition of reference intervals. Clin Chem. 2015;61(5):760-8.

\section{Publisher's Note}

Springer Nature remains neutral with regard to jurisdictional claims in published maps and institutional affiliations.

Ready to submit your research? Choose BMC and benefit from:

- fast, convenient online submission

- thorough peer review by experienced researchers in your field

- rapid publication on acceptance

- support for research data, including large and complex data types

- gold Open Access which fosters wider collaboration and increased citations

- maximum visibility for your research: over $100 \mathrm{M}$ website views per year

At $\mathrm{BMC}$, research is always in progress.

Learn more biomedcentral.com/submissions 